\title{
MODELING GIANT EXTRASOLAR RING SYSTEMS IN ECLIPSE AND THE CASE OF J1407B: SCULPTING BY EXOMOONS?
}

\author{
M. A. Kenworthy ${ }^{1}$ and E. E. MamajeK ${ }^{2}$ \\ ${ }^{1}$ Leiden Observatory, Leiden University, P.O. Box 9513, 2300 RA Leiden, The Netherlands \\ ${ }^{2}$ Department of Physics and Astronomy, University of Rochester, Rochester, NY 14627-0171, USA \\ Received 2014 November 8; accepted 2014 December 28; published 2015 February 18
}

\begin{abstract}
The light curve of 1SWASP J140747.93-394542.6, a 16 Myr old star in the Sco-Cen OB association, underwent a complex series of deep eclipses that lasted 56 days, centered on 2007 April. This light curve is interpreted as the transit of a giant ring system that is filling up a fraction of the Hill sphere of an unseen secondary companion, $\mathrm{J} 1407 \mathrm{~b}$. We fit the light curve with a model of an azimuthally symmetric ring system, including spatial scales down to the temporal limit set by the star's diameter and relative velocity. The best ring model has 37 rings and extends out to a radius of $0.6 \mathrm{AU}\left(9 \times 10^{7} \mathrm{~km}\right)$, and the rings have an estimated total mass on the order of $100 M_{\text {Moon }}$. The ring system has one clearly defined gap at $0.4 \mathrm{AU}\left(6.1 \times 10^{7} \mathrm{~km}\right)$, which, we hypothesize, is being cleared out by a $<0.8 M_{\oplus}$ exosatellite orbiting around J1407b. This eclipse and model imply that we are seeing a circumplanetary disk undergoing a dynamic transition to an exosatellite-sculpted ring structure, which is one of the first seen outside our solar system.
\end{abstract}

Key words: eclipses - planets and satellites: rings - protoplanetary disks - stars: individual (1SWASP J140747.93-394542.6) - techniques: high angular resolution

\section{INTRODUCTION}

Circumstellar disks of gas and dust are a ubiquitous feature of star formation. Circumstellar gas-rich disks disperse on timescales of $<10 \mathrm{Myr}$, effectively limiting the runaway growth phase for gas giant planets (Williams \& Cieza 2011). The architecture of the resultant planetary systems is dictated by the structure and composition of the disk, its interaction with the young star, and the competing formation mechanisms that transfer circumstellar material onto accreting protoplanets (e.g., see reviews by Armitage 2011; Kley \& Nelson 2012). Extended month- to year-long eclipses indicate the presence of longlived dark disks around secondary companions, including $\epsilon$ Aurigae (Guinan \& Dewarf 2002; Kloppenborg et al. 2010), EE Cep (Mikolajewski \& Graczyk 1999; Graczyk et al. 2003; Mikolajewski et al. 2005), a precessing circumbinary disk around KH 15D (Hamilton et al. 2005; Winn et al. 2006), and three systems recently discovered in the OGLE database: OGLE-LMC-ECL-17782 (Graczyk et al. 2011), OGLE-LMCECL-11893 (Dong et al. 2014), and OGLE-BLG182.1.162852 (Rattenbury et al. 2014).

Gas planets are thought to form through accretion from circumstellar disks composed of gas and dust. Angular momentum of the circumstellar disk material is redistributed through the formation of a circumplanetary disk. After the gas is cleared out of the planetary system, dust in the circumplanetary disk then accretes into moons or remains as a ring system within the Roche limit of the planet (Canup \& Ward 2002; Magni \& Coradini 2004; Ward \& Canup 2010). The transits of giant planets with ring systems produce a distinct and detectable light curve (Barnes \& Fortney 2004; Tusnski \& Valio 2011), and searches for the transit timing variations caused by attendant exomoons are ongoing (Kipping et al. 2012, 2013). 1SWASP J140747.93394542.6 (hereafter J1407) is a pre-main-sequence, $\sim 16 \mathrm{Myr}$ old, $0.9 M_{\odot}, V=12.3 \mathrm{mag} \mathrm{K} 5 \mathrm{star}$ at $133 \mathrm{pc}$ associated with the Sco-Cen OB association (Mamajek et al. 2012; van Werkhoven et al. 2014; Kenworthy et al. 2015). ${ }^{3}$ The Super Wide Angle Search for Planets (SuperWASP) database (Butters et al. 2010) shows that the star underwent a complex series of eclipses lasting 56 days around 2007 May and includes a dimming of $>95 \%$. Mamajek et al. (2012) and van Werkhoven et al. (2014) propose that these eclipses are caused by a large ring system orbiting an unseen substellar companion, dubbed J1407b. In the first attempt to model the system using nightly averaged photometry, Mamajek et al. (2012) posited at least four large rings girding J1407b. A more detailed analysis of the SuperWASP raw data by van Werkhoven et al. (2014) and removal of a 0.1 mag amplitude, 3.2 day periodic variability due to rotational modulation by star spots show temporal structure down to a limit of 10 minutes (van Werkhoven et al. 2014). Only the 2007 eclipse event is seen in the time series photometry, and Kenworthy et al. (2015) place constraints on the possible mass and orbital period for this companion. They conclude that $\mathrm{J} 1407 \mathrm{~b}$ is almost certainly substellar (at $>3 \sigma$ significance), and possibly an exoplanet.

The analysis of eclipse light curves to determine the structure of otherwise unresolved astrophysical objects is possible for specific cases. Since the first proposal in MacMahon (1908), high-speed photometry of lunar occultations has been used (White 1987) to determine the multiplicity of stars close to the ecliptic, the structure of evolved stars, and studies of the Galactic center. The sharp inner ring edge of Saturn's Encke gap has been used to deduce the wavelength-dependent radius of Mira using high-speed photometry from Cassini (Stewart et al. 2013). The inverse problem of determining the extended structure of a foreground object assuming a point-like background source has been used to discern fine structures in the rings of the gas giant planets, to measure the scale heights of the atmospheres in planetary bodies such as Titan and Pluto (e.g., McCarthy et al. 2008), and to determine the shape and orbital properties of

\footnotetext{
http://exoplanet.eu/catalog/1swasp_j1407_b/
} 
solar system asteroids (e.g., Dunham et al. 1990; Shevchenko $\&$ Tedesco 2006). The Uranian ring system was discovered by stellar occultation (Elliot et al. 1977, Millis et al. 1977), and more recently, two rings were discovered around an asteroid in the solar system (Braga-Ribas et al. 2014), where the structure in the rings themselves is unresolved. In this paper we use knowledge of an extended background source and multiple-ring structure around a foreground object to derive the geometric properties of the $\mathrm{J} 1407 \mathrm{~b}$ ring system, a candidate circumplanetary disk.

In Section 2 we present our exoring model and then discuss several features to be expected in a ring system transit where the ring system is significantly larger than the parent star. In Section 3 we present our best fits to the J1407b transit data, and in Section 4 we discuss the structure in the most plausible ring models and posit that they are indirect evidence for exomoons or exosatellites coplanar with the rings. The large size of the ring system presents a challenge to what type of orbit it must have around the primary star, which we address in Section 5. Our conclusions are presented in Section 6.

\section{RING MODEL}

Our ring model is composed of two parts, which we solve sequentially for an observed transiting ring system. We assume that the primary star and ring system are at a similar distance from the Earth, and that the ring system is at least several times larger than the angular size of the primary star. We first solve for the orientation of the plane of the ring system relative to our line of sight, and we then solve for the transmission of the rings as a function of radius from the secondary companion, given the geometry of the ring system derived in the previous step.

\subsection{Input Parameters}

The primary is a star at a distance of $d$ parsecs, with radius $R_{\star}$. We approximate the orbit of the secondary for the duration of the eclipse as being a straight line, with constant relative velocity of $v$. Surrounding the secondary companion is a ring system in a plane that contains both the rings and the equatorial plane of the secondary companion, which we refer to as the ring plane. The rings are composed of individual particles that orbit the secondary companion in Keplerian orbits, assumed circular. These particles scatter light out of any incident beam and in aggregate are approximated by a smooth screen with an optical transmission of $\tau(r)$ that varies as a function of radial distance $r$ from the center of the secondary companion. The rings are assumed to be azimuthally symmetric; the inclination of the ring plane as seen from the Earth is $i_{\text {disk }}$ (with $0^{\circ}$ being face-on), and the projected angle between the normal of the secondary companion's orbit and the normal of the ring plane is $\phi_{\text {disk }}$. The obliquity $\varepsilon$ of the ring plane is related to these two angles by

$$
\cos \varepsilon=\sin i_{\text {disk }} \cos \phi_{\text {disk }} \text {. }
$$

The rings are assumed to be considerably thinner than their diameter, i.e., a "thin ring" approximation. In Saturn's ring system, thicknesses from tens of meters down to an instrumentresolved limit of tens of centimeters (Tiscareno 2013) have been observed. Pole-on, the rings form a concentric set of circles centered on the secondary companion. The light curve of a point source passing behind the ring structure along an arbitrary chord is therefore symmetric in time about the point of closest approach of the projected star position to the companion. What may not be so obvious is that a thin ring system tilted at an arbitrary inclination will also produce a symmetric light curve, regardless of the chord chosen. This can be seen when one considers that a set of concentric ellipses can be transformed into a set of concentric circles by a single shear transformation whose shear axis is parallel to the chord.

The light curve $I(t)$ of a source with finite angular size (i.e., the stellar disk of the primary) behind a tilted ring system, however, is not time symmetric (see Figure 1). For each ring boundary, the gradient of the light curve $g(t)$ is dependent on both the size of the star and the angle between the local tangent of the ring edge and the direction of motion. Ring structures smaller than that of the stellar diameter are smeared out by the resultant convolution with the stellar disk, resulting in a characteristic timescale defined by the time taken for the stellar disk to cross its own diameter $t_{\star}=2 R_{\star} / v$.

The track of the star on the projected ring plane has its closest approach at time $t_{b}$ with an impact parameter of $b$, along with the ring orientation defined by $i_{\text {disk }}$ and $\phi_{\text {disk }} \cdot i_{\text {disk }}$ is the inclination of the plane of the rings to the plane of the sky. $\phi_{\text {disk }}$ is the rotation of the ring system in the plane of the sky in an anticlockwise direction. These four parameters uniquely define the relationship between epoch of observation $t$ and ring radius $r$. We model an azimuthally symmetric ring of radius $r$ seen in projection by an ellipse with the secondary companion at the origin with a semimajor axis $r$ and semiminor axis $r \cos i$. The semimajor axis of the ellipse is rotated anticlockwise from the $x$-axis by an angle $\phi$. The parametric equation for a projected ring is then

$$
\begin{aligned}
& x(p)=r\left(\cos p \cos \phi_{\text {disk }}-\sin p \cos i_{\text {disk }} \sin \phi_{\text {disk }}\right), \\
& y(p)=r\left(\cos p \sin \phi_{\text {disk }}+\sin p \cos i_{\text {disk }} \cos \phi_{\text {disk }}\right),
\end{aligned}
$$

where $x$ and $y$ are coordinates on the ring at radius $r$ at a given value of the parametric variable $p$, which has a value from 0 to $2 \pi$ radians.

The star moves along a line parallel to the $x$-axis:

$$
\begin{aligned}
& x_{\star}=v\left(t-t_{b}\right) \\
& y_{\star}=b
\end{aligned} .
$$

In addition to the time of closest projected approach of the star to the secondary companion, there are two other significant epochs: the epoch when the stellar motion is perpendicular to the ring-projected ellipse $t_{\perp}$, and the epoch $t_{\|}$when the stellar motion is tangential to the ring-projected ellipse.

To find where the tangent of the ring is perpendicular to the $y$-axis, we see where $d x / d p=0$. The result is then

$$
\tan p_{\perp}=-\cos i_{\text {disk }} \tan \phi_{\text {disk }}
$$

Since nested rings only differ in a single scale factor centered on the origin, the loci of all perpendicular tangents lie on a straight line passing through the origin, i.e., the geometric center of the rings. The angle $\theta_{\perp}$ can be calculated by substitution:

$$
\tan \theta_{\perp}=\frac{y\left(p_{\perp}\right)}{x\left(p_{\perp}\right)} .
$$

A similar derivation gives the angle for the line passing through the loci of all parallel tangents, $\theta_{\|}$:

$$
\tan p_{\|}=\left(\cos i_{\text {disk }} \tan \phi_{\text {disk }}\right)^{-1}
$$




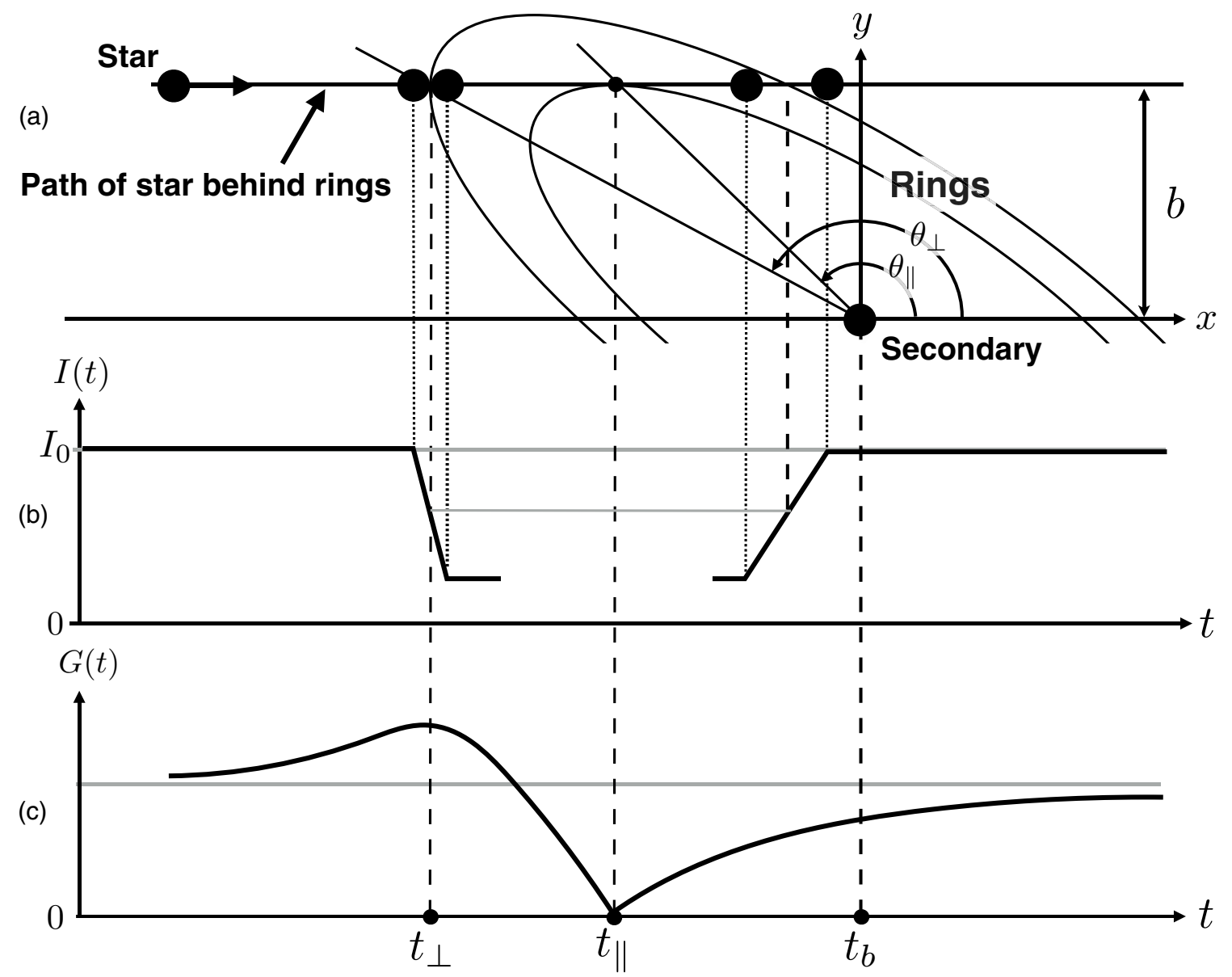

Figure 1. Geometry of the ring model. Panel (a) shows a ring system inclined at an angle of $i_{\text {disk }}$ and rotated from the line of relative velocity by $\phi_{\text {disk }}$. The star passes behind the ring system with impact parameter $b$ at time $t_{b}$. Panel (b) shows the resultant light curve $I(t)$ of the star as a function of time, demonstrating how the local ring tangent convolved with the finite-sized disk of the star produces light curves with different local slopes. Panel (c) highlights the three significant epochs in the rate of change of ring radius $r: t_{b}$, at closest projected separation of the star and the secondary; $t_{\perp}$, where the ring tangent is perpendicular to the direction of stellar motion; and $t_{\|}$, where stellar motion is tangent to the ring. $t_{\|}$also marks where the stellar path touches the smallest ring radius.

and

$$
\tan \theta_{\|}=\frac{y\left(p_{\|}\right)}{x\left(p_{\|}\right)}
$$

Finally, for distances where the star/secondary companion distance is much larger than the impact parameter (i.e., where $x$ tends to very large values with $y=0$ ), the gradient $d y / d x$ tends toward an asymptote defined by

$$
\tan \theta_{y=0}=\left.\frac{d y}{d x}\right|_{y=0}=\frac{2\left(\sin ^{2} \phi_{\text {disk }}+\cos ^{2} \phi_{\text {disk }}\right)}{\sin ^{2} \phi_{\text {disk }} \sin ^{2} i_{\text {disk }}} .
$$

These three regimes are shown in the lower panel of Figure 1 as the time of largest gradient, the gradient touching at zero, and the asymptotic gradient at large positive and negative values along the $x$-axis.

A simple ring model is uniquely defined with four numbers: the orientation of the ring system (the inclination and obliquity), the impact parameter $b$, and the epoch of closest projected approach to the secondary companion $t_{b}$. The transmitted intensity of light through a ring at radius $r$ with $\tau(r)$ is

$$
I(r)=I_{0} e^{-\tau(r)} .
$$

Since we do not know whether the rings are a single thin screen of particles or are optically thick, we do not correct $\tau$ for the inclination of the ring system.
The greatest uncertainty in the model fitting is the diameter of the star. We therefore express the size of the rings in units of time, converting back to linear sizes at the end of the modeling. With an assumed stellar size and relative velocity, the system can be converted back into units of length by multiplying by $v$. A light-curve model for a given star is produced with the diameter of the star, its relative velocity with respect to the secondary companion, the limb-darkening parameter, the orientation, and the radial transmission of the ring system.

\section{THE LIGHT CURVE OF J1407}

The complex light curve of J1407 was first discussed in Mamajek et al. (2012), where intensity fluctuations of up to 95\% were seen over a 56-day period in 2007 April and May toward this young ( $\sim 16 \mathrm{Myr}) \mathrm{K} 5$ pre-main-sequence star in photometry taken as part of the SuperWASP Survey (Pollacco et al. 2006; Butters et al. 2010). After ruling out other simpler astrophysical explanations, Mamajek et al. (2012) concluded that the light curve was due to the transit of a giant ring system orbiting an unseen secondary companion, and that this ring system was considerably larger than the diameter of the central star. The star was simultaneously observed by three of the eight cameras in the SuperWASP South array, and owing to its location in the corner of the field of view of these three cameras, there is a clear systematic offset of 
0.3 mag seen between the cameras in the standard data reduction photometry. A dedicated reprocessing of all the raw photometric data successfully removes both the systematic offsets seen in the light curves and also the much smaller amplitude stellar variability (van Werkhoven et al. 2014). We use the cleaned photometric data (van Werkhoven et al. 2014) as the input for our ring fitting model. The observations over the 54-day period are not continuous but are interrupted by the diurnal cycle and cloud cover at the observing site, resulting in a completeness of $11.3 \%$.

Fitting is performed in a two-step process: we first constrain the orientation of the ring system using the gradients measured from the light curve (Section 3.1), and then we use these parameters to generate a model of the ring transmission as a function of radius from the secondary companion (Section 3.2).

The angular diameter of the star is $65.2 \pm 9.3 \mu$ as, based on a distance $d=133 \pm 12 \mathrm{pc}$ and radius of $0.99 \pm 0.11 R_{\odot}$ (van Werkhoven et al. 2014; Kenworthy et al. 2015). Treating a transiting ring as a semi-infinite knife edge, and assuming a point source behind the rings, the angular separation between the geometric edge of the ring shadow and the first diffraction maximum is $1.22 \sqrt{ } \lambda / 2 d$, giving an angular fringe separation at $62 \pm 4$ nas, about 1000 times smaller than the angular diameter of the star. Using geometric shadows is therefore a valid approximation for the model.

\subsection{Fitting the Ring Orientation Using Light-curve Gradients}

For a given set of ring orientation parameters $i_{\text {disk }}, \phi_{\text {disk }}, b, t_{b}$, we can determine the radial distances of the rings from the secondary companion (i.e., the ring radius) at any epoch, $r(t)=f\left(i_{\mathrm{disk}}, \phi_{\mathrm{disk}}, b, t_{b}, t\right)$, and also determine $d r(t) / d t$. The transmission of the disk as a function of $r$ is given by $\tau(r)$. Together with a model of the stellar disk that includes limb darkening (see van Werkhoven et al. 2014) and the functional form of $\tau(r)$, we can calculate the light curve of a ring system model for any epoch. In practice, we calculate a grid of values of $r$ for the track of the star behind the ring system, with a spatial resolution set by the diameter of the star. The transmission $I(r)$ is calculated for all points in the grid along the track. This grid of flux values is then convolved with a model of the stellar disk, and the line of pixels along the impact parameter $b$ then represents the measured flux $I(t)$. We use 25 pixels across the diameter of the star to sample the limb darkening and stellar disk.

The measured flux $I(t)$ of J1407 can be closely approximated as a sequence of straight lines of different gradients (see van Werkhoven et al. 2014, for details). We interpret these straightline light curves as a ring edge (between two rings with different values of transmission) passing across the disk of the star. When the slope of the light curve changes, this represents a ring edge either starting or finishing its transit of the stellar disk. The measured gradients of straight-line fits to the J1407 light curve are shown as the black circles in Figure 3. We assume that all the rings have well-defined edges and have a constant transmission across the width of the ring (with some assumed constant $\tau$ ); see Figure 2.

To understand our ring orientation algorithm, consider a ring system made up of alternately transparent and opaque rings whose radial width would allow complete obscuration or transmission of the stellar disk. The transmitted intensity goes from $I=I_{0}$ to $I=0$ and vice versa at a rate determined by the ring velocity $v$ and the local tangent of the ring to the line of stellar motion, defined as parallel to the $x$-axis in our model. If the gradient of the light curve is measured close to the midpoint

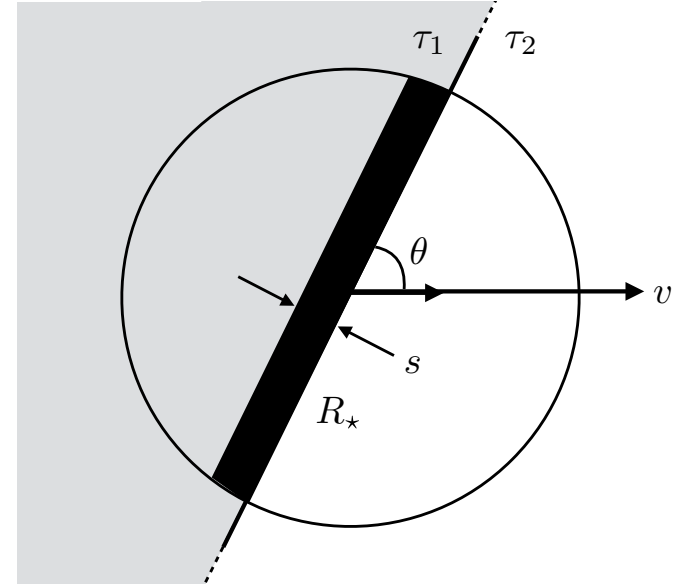

Figure 2. Geometry of a ring edge crossing a stellar disk of uniform intensity. The stellar disk is of uniform illumination with radius $R_{\star}$, and we do not show limb darkening in this example. The ring edge moves at a velocity $v$ across the disk. The ring edge is at an angle $\theta$ to the direction of motion $v$. The black strip has a width of $s$. The two rings have absorption coefficients of $\tau_{1}$ and $\tau_{2}$.

of the transit of a ring edge and this quantity is plotted as a function of time, the result is the black curve in Figure 3.

Defining the angle between the tangent of the ring at point $\left(x_{\star}, y_{\star}\right)$ and the $x$-axis as $\theta$ (see Figure 2),

$$
\tan \theta=d y / d x \text {. }
$$

The area swept out by a straight edge in time $d t$ across a stellar disk with no limb darkening is equal to $2 R_{\star} s$, where $s=$ $v d t \sin \theta$. Defining the transmission $T_{n}=I_{n} / I_{0}=\exp \left(-\tau_{n}\right)$, the change in intensity $d I$ is simply the change in transmission from $T_{1}$ to $T_{2}$ over the area $2 R_{\star} v \sin \theta d t$, so the rate of change of intensity is

$$
\begin{aligned}
g(t) & =\frac{d I(t)}{d t}=\left(T_{1}-T_{2}\right) G(t) \\
& =\left(T_{1}-T_{2}\right) \frac{2 v \sin \theta}{\pi R_{\star}}\left(\frac{12-12 u+3 \pi u}{12-4 u}\right) .
\end{aligned}
$$

The limb darkening of the star is parameterized by $u=0.8(5)$ for J1407 (Claret \& Bloemen 2011).

The function $G(t)$ represents the maximum flux change possible between a fully transparent and fully opaque ring. For rings that have intermediate values of transmission, the resultant light gradients will lie underneath this black curve, and so the curve represents an upper bound on the light gradient for a given ring orientation. Since we do not know $\tau(r)$, we can use $G(t)$ as an upper limit, and we search for ring orientations that have all measured gradients lying underneath this curve.

We have $n$ measurements of the light-curve gradient $g(t)$ at time $t$. The model gradient $G(t)$ is calculated from Equation (1), where $\theta$ is a function of $i_{\text {disk }}, \phi_{\text {disk }}, b, t_{b}, t$. We calculate a cost function that minimizes the difference between the model and measured gradients and penalizes heavily if the measured point goes above the model point. If we define $\delta_{t}=G(t)-g(t)$, then our cost function $\Delta$ is

$$
\Delta=\sum_{t=1}^{n}\left\{\begin{aligned}
\delta_{t} & \text { if } \delta_{t}>0 \\
-50 * \delta_{t} & \text { otherwise. }
\end{aligned}\right.
$$

The factor of 50 used in the above equation reflects the error of $2 \%$ on the measured light-curve gradients and prevents fitting algorithms from oscillating near local minima. We use an 


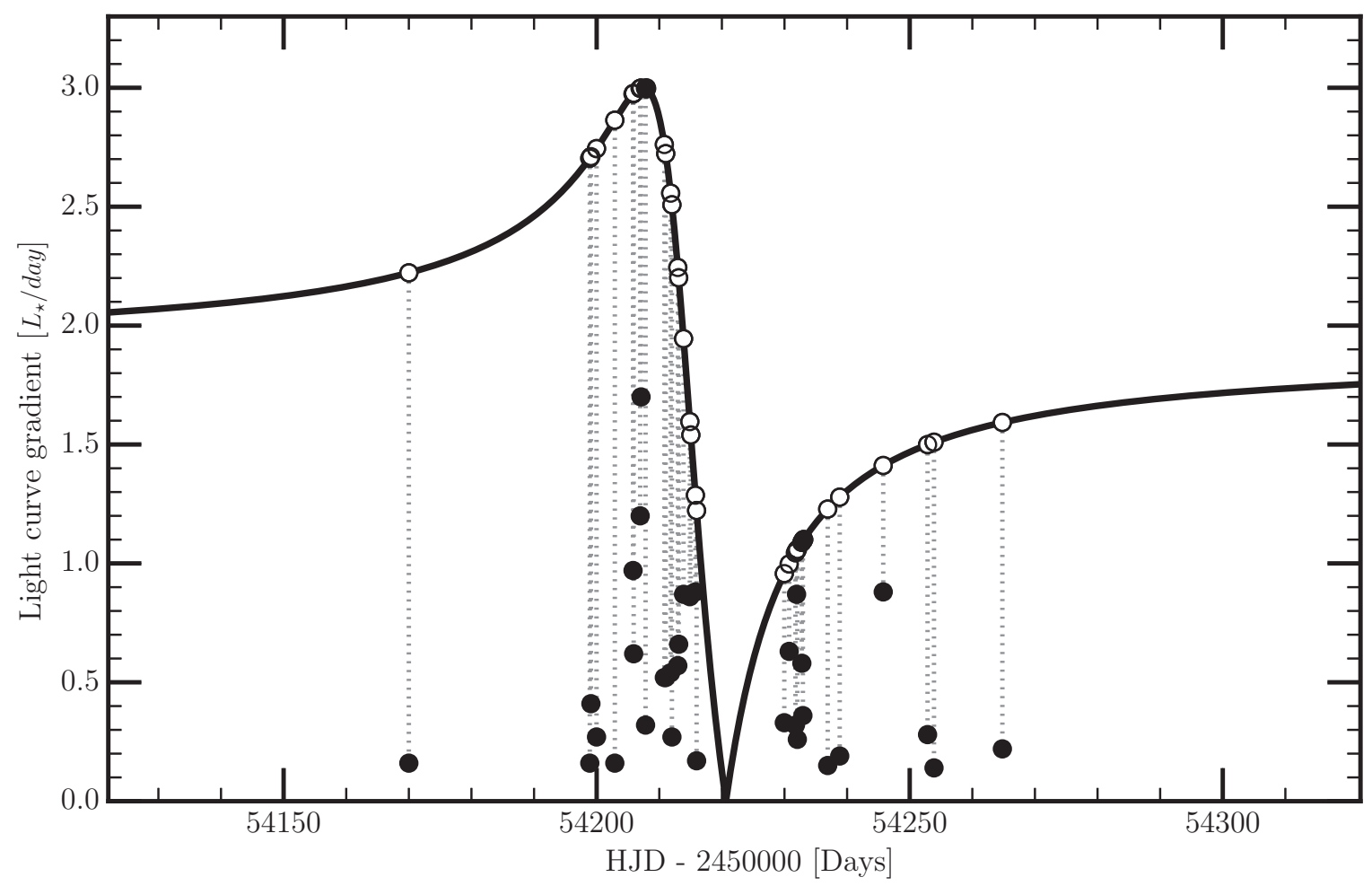

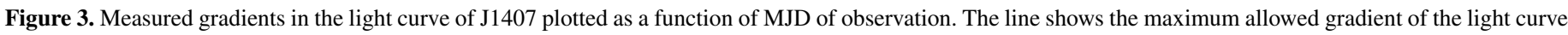

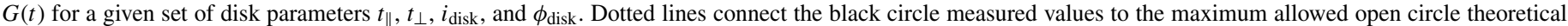
maximum values on the black line.

Table 1

Disk Model Parameters

\begin{tabular}{ccccccc}
\hline \hline Model & $\begin{array}{c}b \\
(\text { days })\end{array}$ & $\begin{array}{c}t_{b} \\
(\text { days })\end{array}$ & $\begin{array}{c}i_{\text {disk }} \\
(\mathrm{deg})\end{array}$ & $\begin{array}{c}\phi_{\text {disk }} \\
(\mathrm{deg})\end{array}$ & $\begin{array}{c}t_{\|} \\
(\text {days })\end{array}$ & $\begin{array}{c}v \\
\left(\mathrm{~km} \mathrm{~s}^{-1}\right)\end{array}$ \\
\hline 1 & 3.92 & 54225.46 & 70.0 & 166.1 & 54220.65 & 33.0 \\
\hline
\end{tabular}

Amoeba simplex algorithm (Press et al. 1992) to solve for the four free parameters. The resulting behavior of the cost function is to bring down the model curve $G(t)$ so that at least two measured gradient points from $g(t)$ lie on $G(t)$, which may not be necessarily correct if the data are sparse and the measured slopes do not sample the largest gradient of $G(t)$ at $t_{\perp}$. In the case of J1407, the largest observed gradient is during MJD 54,220, and the complete set of parameters for the disk geometry is listed in Table 1. It is highly probable that this is the largest gradient in the ring system since the secondary companion velocity derived from this gradient presents a challenge to the orbital dynamics for rings (see van Werkhoven et al. 2014; Kenworthy et al. 2015, for a detailed discussion). We therefore introduce an additional cost function that fixes the midpoint of the eclipse light curve $t_{\perp}$ to a user-defined value, so that we can explore different ring geometries that still produce reasonable cost functions.

The minimum velocity required to cross a limb-darkened star is derived in van Werkhoven et al. (2014), and for the case of $\mathrm{J} 1407$, Equation (12) gives a relative velocity of $33 \mathrm{~km} \mathrm{~s}^{-1}$ for $R_{\star}=0.99 R_{\odot}$ and $\dot{L}_{\max }=3.1 L_{*}$ day $^{-1}$. We adopt these values for our model.

\subsection{Fitting the Ring Structure}

The ring radius $r(t)$ can be calculated with values for $i_{\text {disk }}, \phi_{\text {disk }}, b, t_{b}$ estimated from the disk fitting procedure of the previous section. We now look for the ring transmission as a function of radius by using our model of $r(t)$, the stellar radius and limb-darkening profile of $\mathrm{J} 1407$, and an estimate of the transverse velocity $v$. We adopt the limb-darkening profile and parameters of van Werkhoven et al. (2014) and a stellar radius of $0.99 R_{\odot}$ (Kenworthy et al. 2015). The number of ring edges in the light curve is estimated by counting the number of slope changes identified in the light curve and indirectly implied by the change of the light curve during daylight hours. At least 24 ring edges are required for the number of gradient changes detected in the J1407 data (van Werkhoven et al. 2014), but given the sparseness of the photometric coverage, this number is almost certainly higher.

Using the derived ring geometry parameters, the absolute value of the time since the closest approach of the secondary companion, abs $\left(t-t_{\|}\right)$, is used as the origin for a graph that displays the observed light curve, the model light curve, and the difference of these two curves. Displaying the data and model in this way allows a direct visual comparison between the ingress and the egress of the star around the time of closest projected ring approach of the secondary companion $t_{\|}$. In the limiting case of a point-like background source, the ingress light curve and egress light curve are identical for azimuthally symmetric ring systems. The finite diameter of the star breaks this degeneracy, and so the ingress and egress light curves for a given ring edge radius are not identical. We generate an initial estimate of $\tau(r)$ using a GUI written in the Python programming language. This is defined as

$$
\tau(r)=\tau_{n} \text { for } r_{n-1}<r<r_{n} \text { with } r_{0}=0 \text { and } n=1 \text { to } N_{\text {rings }} \text {. }
$$

Ring edges and transmission values are interactively added, moved, and deleted as appropriate, and the ring model $I(t)$ is generated after each successive operation. A visual inspection of the model light curve and its comparison to the data is carried out, and when the minimum number of rings is added to the 

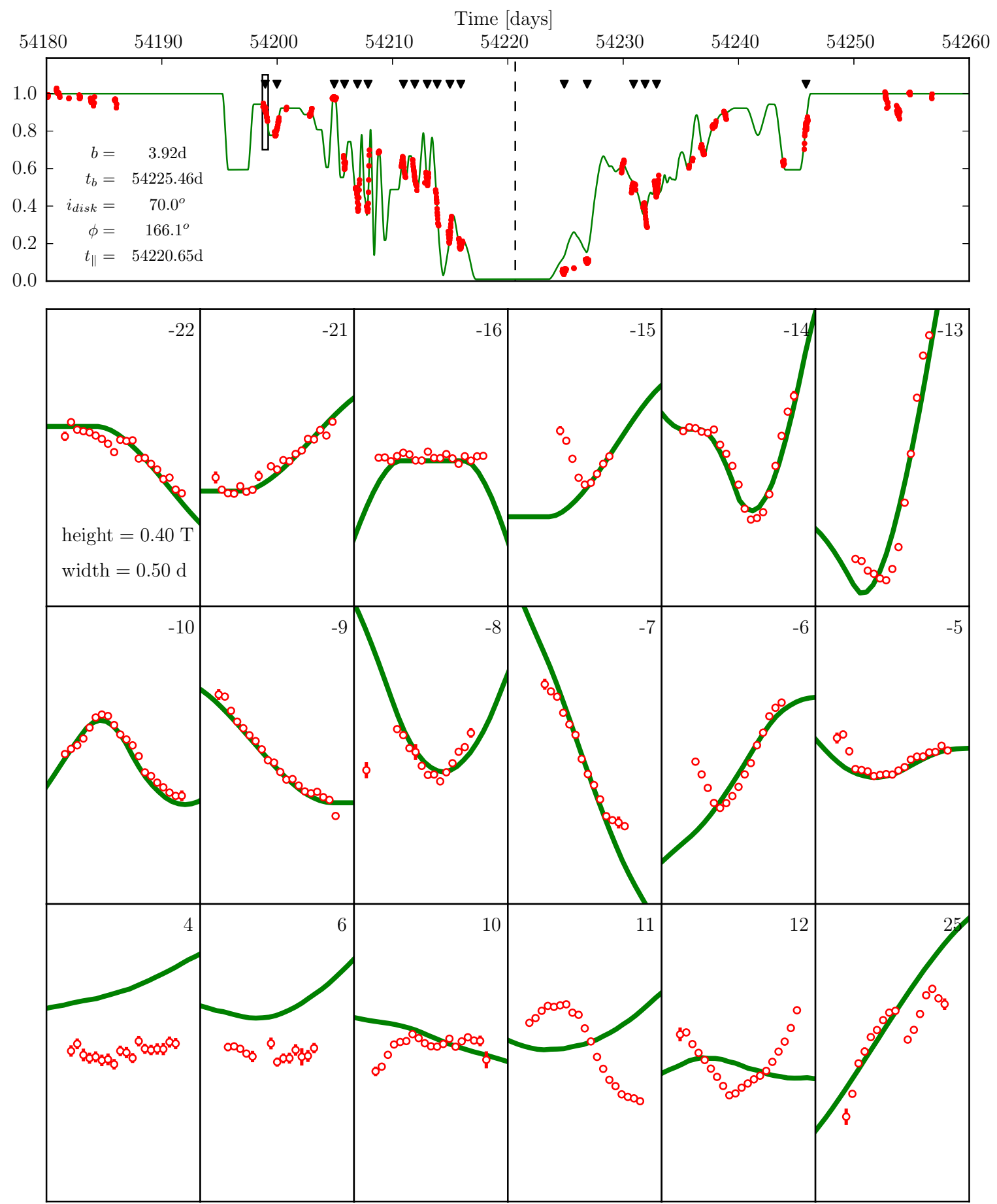

Figure 4. Photometry of J1407. The upper panel shows the light curve of J1407 as the red points with associated error bars. The green curve is the model fit for Model 1, with $t_{\|}=54,220.65$ MJD (indicated with the vertical dashed line) and $v=33 \mathrm{~km} \mathrm{~s}^{-1}$. For the nights indicated with the inverted triangles, the photometry and model fit are enlarged into the panels below. Each panel has a width of 0.5 days and a height of 0.4 in transmission. The number in the top right corner represents the number of days from $t_{\|}$.

model, the ring transmission values are optimized using a minimum least-squares fit to the data and Amoeba simplex algorithm.

There are 16,489 photometric data points covering the 2007 observing season of J1407 (MJD 54,131.96 to 54,306.72). The data of J1407 do not show significant changes in flux photometry over the timescale of 30 minutes, and so to speed up the interactive fitting, the photometric data are rebinned in 0.02-day (32-minute) intervals with error estimates calculated from the rms of the photometric data points within each bin. Bins containing less than three photometric points are discarded, resulting in a data series of 985 points. The photometric data are sparse (the binned data cover $11.3 \%$ of the total 2007 season), and so no unique solutions for the disk geometry and $\tau(r)$ are found. ${ }^{4}$

In Figures 4 and 5 we present one possible ring solution (listed as Model 1 in Table 1) to the J1407 photometric data, where the central eclipse is set at $t_{b}=54,220.65 \mathrm{MJD}$.

\footnotetext{
4 When the light curve is folded at time of closest approach, the coverage approximately doubles to $20 \%$. This implies that there are at least $24 / 0.2 \approx 120$ ring edges in the J1407b system.
} 


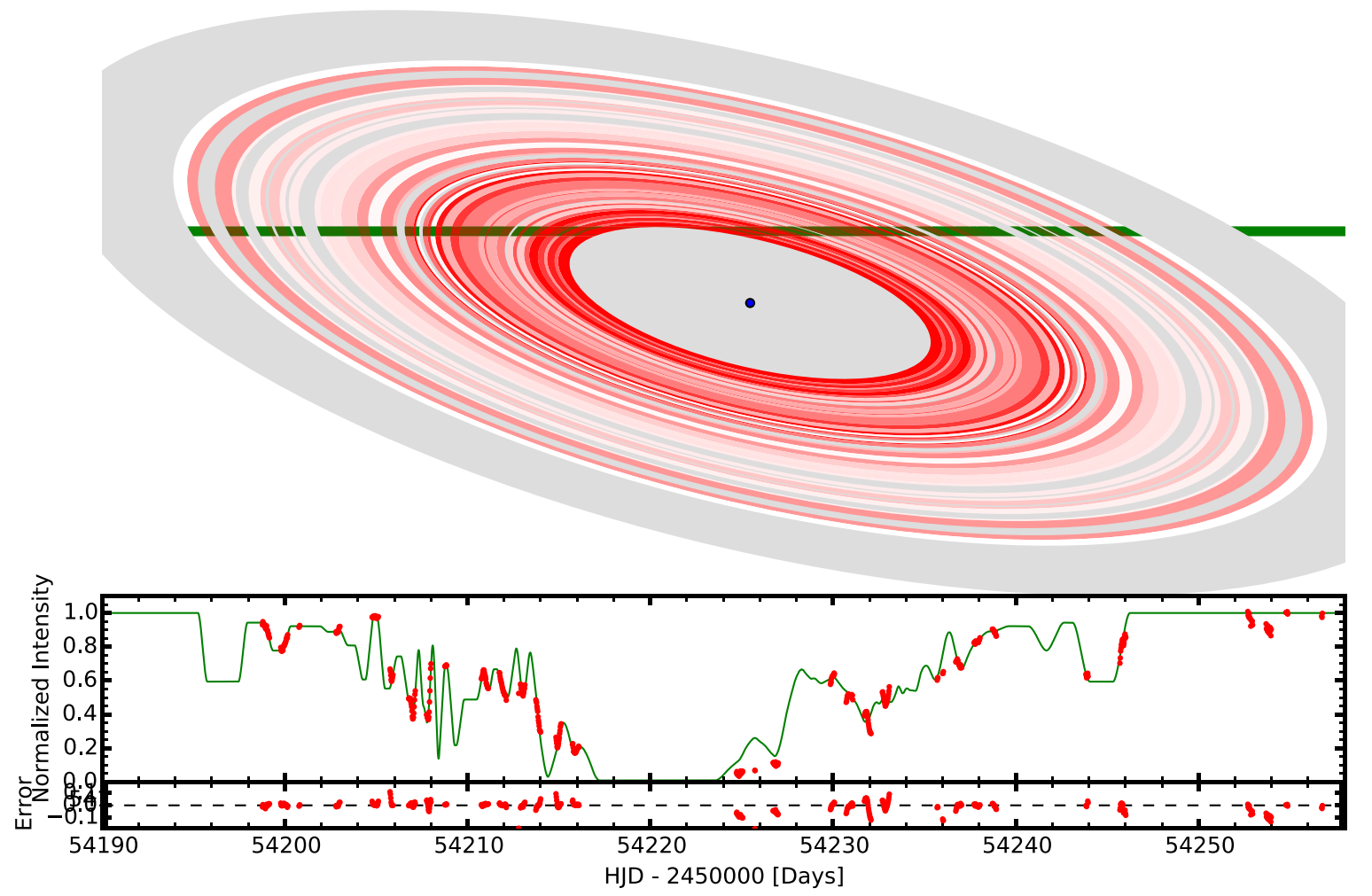

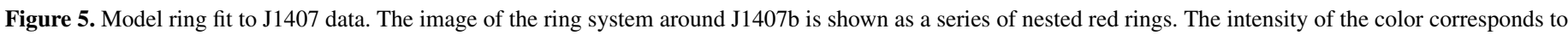

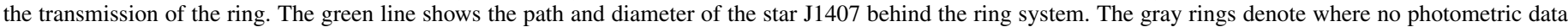

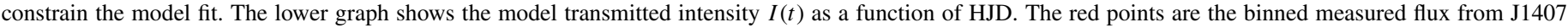
normalized to unity outside the eclipse. Error bars in the photometry are shown as vertical red bars.

\section{INTERPRETING THE J1407B MODEL}

By visual examination there is a clear decrease and subsequent increase in the transmission of $\mathrm{J} 1407$ flux with a minimum around MJD 54,220. Using photometry averaged in $24 \mathrm{hr}$ bins, a ring model with four broad rings is consistent with data on these timescales (Mamajek et al. 2012). On hourly timescales, the large flux variations are consistent with sharp-edged rings crossing over the unresolved stellar disk. We do not find an azimuthally symmetric ring model fit that is consistent with all the photometric data at these timescales. Owing to the incomplete photometric coverage, there are several models that fit with similar $\chi^{2}$ values to the data. In all of these cases, we see the presence of rapid fluctuations in the ring transmission both as a function of time and as a function of radial separation from the secondary companion.

There are clear gaps in all the ring model solutions explored. Gaps in the rings of solar system giant planets are caused either directly by the gravitational clearing of a satellite or indirectly by a Lindblad resonance due to a satellite on a larger orbit. The $\mathrm{J} 1407$ ring system is larger than its Roche limit for the secondary companion. A search for the secondary companion is detailed in Kenworthy et al. (2015), and the constraints from null detections in a variety of methods result in a most probable mass and orbital period for the secondary companion. These orbital parameters are summarized in Table 3. We take the most probable mass and period for the moderate range of eccentricities with mass $23.8 M_{\text {Jup }}$ and orbital period $13.3 \mathrm{yr}$, although we note that the period could be as short as $10 \mathrm{yr}$ and the mass can be greater than $80 M_{\text {Jup }}$, but this mass is considered highly unlikely, with a probability of less than $1.2 \%$. Gaps in the ring system are seen either directly as the photometric flux from J1407 returning to full transmission during the eclipse or indirectly as a fit of the model to intermediate transmission photometric gradients. One ring gap with photometry is at HJD 54,210, seen during the ingress of J1407 behind the ring system. The corresponding radius for this gap in the disk is seen from $5.9 \times 10^{7} \mathrm{~km}$ to $6.3 \times 10^{7} \mathrm{~km}$ (indicated in Figure 6), corresponding to an orbital period $P_{\text {sat }}$ of

$$
P_{\text {sat }}=1.7 \mathrm{yr}\left(\frac{M_{J 1407 b}}{23.8 M_{\text {Jup }}}\right)^{-1 / 2}
$$

If we assume that the gap is equal to the diameter of the Hill sphere of a satellite orbiting around the secondary companion and clearing out the ring, then an upper mass for a satellite can be calculated from

$$
m_{\mathrm{sat}} \approx 3 M_{b}\left(\frac{d_{\mathrm{hill}}}{2 a}\right)^{3}=0.8 M_{\oplus}\left(\frac{M_{J 1407 b}}{23.8 M_{\mathrm{Jup}}}\right) .
$$

For the case of $23.8 M_{\text {Jup }}$ this corresponds to a satellite mass of $0.8 M_{\oplus}$ and an orbital period of $1.7 \mathrm{yr}$.

The ring transmission at smaller radii shows structures that are analogous to the Kirkwood gaps in the solar system, where the smooth radial distribution of asteroids is interrupted by perturbations due to period resonances with Jupiter. In the case of the largest ring around Saturn, the Phoebe ring is not coplanar with the other rings (Verbiscer et al. 2009) but lies in the plane of Saturn's orbit. This is due to the dominance of solar perturbations over the gravitational perturbation caused by the $J_{2}$ contribution of Saturn's gravitational field. The planet $\beta$ Pictoris $\mathrm{b}$ was recently shown to have a rotation period of 


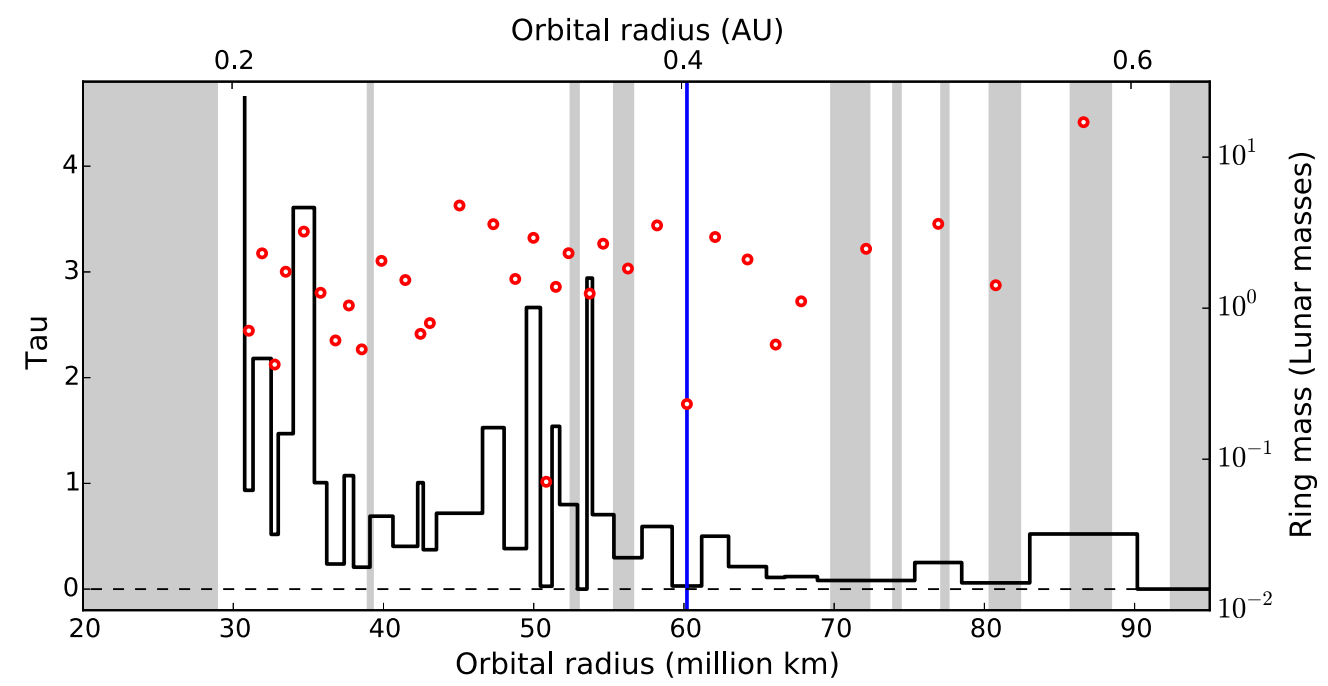

Figure 6. Transmission of the ring model as a function of radius. The gray regions indicate where there is no photometry to constrain the model. The blue line indicates the ring gap seen at $6.1 \times 10^{7} \mathrm{~km}$. Red dots indicate the estimated mass of each ring assuming a mass surface density of $\sim 50 \mathrm{~g} \mathrm{~cm}^{-2}$.

Table 2

Table of Ring Parameters

\begin{tabular}{ll}
\hline \hline $\begin{array}{l}\text { Ring Outer Edge Radius } \\
\left(10^{6} \mathrm{~km}\right)\end{array}$ & Tau \\
\hline 30.8 & 4.65 \\
31.3 & 0.93 \\
32.5 & 2.18 \\
33.0 & 0.52 \\
34.0 & 1.47 \\
35.4 & 3.61 \\
36.2 & 1.01 \\
37.4 & 0.24 \\
38.0 & 1.07 \\
39.1 & 0.21 \\
40.6 & 0.69 \\
42.3 & 0.40 \\
42.6 & 1.01 \\
43.5 & 0.37 \\
46.6 & 0.72 \\
48.0 & 1.53 \\
49.5 & 0.38 \\
50.4 & 2.66 \\
51.2 & 0.03 \\
51.7 & 1.54 \\
52.9 & 0.80 \\
53.5 & 0.00 \\
53.9 & 2.94 \\
55.3 & 0.70 \\
57.2 & 0.30 \\
59.2 & 0.59 \\
61.2 & 0.00 \\
63.0 & 0.50 \\
65.5 & 0.21 \\
66.7 & 0.11 \\
68.9 & 0.12 \\
75.4 & 0.08 \\
78.5 & 0.25 \\
83.0 & 0.06 \\
90.2 & 0.52 \\
\hline & \\
& \\
\hline
\end{tabular}

$\sim 8 \mathrm{hr}$ (Snellen et al. 2014), faster than that of the other gas giants in the solar system. Its age of 22 Myr (Mamajek \& Bell 2014) is similar to the $\mathrm{J} 1407$ system, implying that a low-mass companion could have a fast rotation period, leading to a larger
Table 3

Table of J1407b Orbital Parameters (from Kenworthy et al. 2015)

\begin{tabular}{lcc}
\hline \hline Orbital Eccentricity & $0.7<e<0.9$ & $0.7<e<0.8$ \\
\hline Probable period $\bar{P}(\mathrm{yr})$ & 27.5 & 13.3 \\
Probable mass $\bar{M}\left(M_{\text {Jup }}\right)$ & 14.0 & 23.8 \\
\hline
\end{tabular}

oblateness and corresponding $J_{2}$ contribution, that could hold ring structures in the equatorial plane out to larger radii.

\subsection{Notable Timescales in the Giant Exoring Model}

There are two additional timescales that are worth noting in this giant exoring model. At the time of $t_{\|}$, the star is close to stationary in $r(t)$ and is moving tangentially to the ring at radius $r\left(t_{\|}\right)$. There is therefore a time interval $\Delta t_{1}$ where the star is sensitive to variations in the azimuthal structure around radius $r\left(t_{\|}\right)$. The length of time for this interaction is approximately the length of time it takes for the projected disk radius to change by the diameter of the star, i.e., where

$$
r\left(t_{\|}\right)+\left(R_{*} / \cos i_{\text {disk }}\right)=r\left(t_{\|}+\Delta t_{1}\right) .
$$

This timescale for the $\mathrm{J} 1407$ exoring system is approximately 1 day around $t_{\|}$. We cannot examine the J1407b data for $\Delta t_{1}$ as there is no recorded photometry within 3 days of $t_{\|}$.

The second timescale relates to ring material orbiting around the secondary companion and the duration of the disk transit. If the secondary companion is sufficiently massive and the impact parameter $b$ small enough, there will be a parcel of ring material that will transit in front of the star at least twice. The orbital motion of ring material about the secondary companion, combined with the orbital motion of the secondary companion about the primary star, will result in two epochs symmetric about $t_{\|}$, where the measured transmission will be of the same parcel of ring material. Tests for ring illumination geometry and dust scattering can then be carried out, to name one possibility for this. The timescale for $\mathrm{J} 1407$ is less than 4 days, with a more specific number dependent on disk geometry and secondary mass of the companion. 


\section{THE ORBIT OF J1407B}

Only one eclipse of J1407 is seen in the publically available photometric data, and so we do not know the orbital period of $\mathrm{J} 1407 \mathrm{~b}$ or even whether it is bound to $\mathrm{J} 1407$ in a closed orbit. ${ }^{5}$ We explore two hypotheses for the motion of the ring system relative to the star J1407: (1) the ring system is unbound to J1407 and is a free-floating planet with a ring system, and (2) J1407b is in a bound and closed orbit about J1407. Investigations into the latter case are detailed in van Werkhoven et al. (2014) and Kenworthy et al. (2015).

One estimate of the transverse ring system velocity is calculated from the diameter of the star J1407 and the steepest light-curve gradient seen in the photometric light-curve data. Assuming a sharp-edged opaque ring crossing the disk of the star with the ring edge perpendicular to the direction of motion, a minimum velocity of $33 \mathrm{~km} \mathrm{~s}^{-1}$ is derived (Kenworthy et al. 2015). Combined with the duration of the eclipses, an estimate of the size of the ring system can be made. It is this derived transverse velocity and associated ring system size that form our central issue with the nature of the system.

\subsection{The Ring System is an Unbound Object}

We consider whether the ring system is on an unbound trajectory with a tangential velocity of at least $33 \mathrm{~km} \mathrm{~s}^{-1}$. The 2007 May eclipse is therefore a single event that will not be repeated again with $\mathrm{J} 1407$. Our derived ring model is consistent with the data, yielding a diameter of 1.2 AU for the ring system. We consider the unbound hypothesis as exceptionally unlikely, for two reasons:

1. The mean projected separation between stars in the field is $\approx 10^{3} \mathrm{AU}$. The probability that a $1 \mathrm{AU}$ scale object produces an eclipse within the lifetime of SuperWASP is exceptionally small.

2. J1407b is substellar and almost certainly of planetary mass (Kenworthy et al. 2015). The estimated size of the ring system is two orders of magnitude larger than the Roche limit for the central substellar mass, and so the rings outside the Roche limit are expected to accrete into satellites on timescales shorter then gigayears. This implies that the ring system is considerably younger than stars seen in the field.

Direct imaging limits reported in Kenworthy et al. (2015) constrain such a free-floating object to be $8 M_{\text {Jup }}$, assuming an age of $16 \mathrm{Myr}$ and BT-SETTL models (Allard et al. 2012). We conclude that the ring system is bound to J1407 in a closed orbit.

\subsection{J1407b Is on a Bound Orbit}

In Kenworthy et al. (2015) a search for J1407b is carried out using photometry, radial velocity measurements of J1407, and direct and interferometric techniques. The companion is not detected, but upper limits from these observations constrain the possible orbital period and mass of J1407b. A circular orbit for J1407b would mean that any ring system would not be subject to gravitational perturbations due to the orbit of $\mathrm{J} 1407 \mathrm{~b}$, and so appears preferable for ring stability. Transverse velocities derived from the light-curve gradients, however, strongly rule out long orbital periods, but the lack of a second primary eclipse in time-series photometry rules out short orbital periods.

\footnotetext{
5 By using the name J1407b for the eclipsing object, we are implicitly assuming that the ring system is bound to $\mathrm{J} 1407$ for the reasons expanded on later in this section.
}

A circular orbit is possible if the rings are themselves clumpy in nature and the clump orbital motion vectorially adds to the J1407b orbital motion van Werkhoven et al. (2014), but this again requires a series of coincidences to occur to generate a large gradient at the appropriate epochs.

An elliptical orbit with the eclipse coincident with periastron passage of $\mathrm{J} 1407 \mathrm{~b}$ can provide the transverse velocity required for the ring model. Constraints presented in Kenworthy et al. (2015) suggest a minimum eccentricity of 0.7 and a minimum period of $10 \mathrm{yr}$. The longest period is unconstrained in this model, as very highly eccentric orbits are possible with the long axis pointing toward Earth, but the probability of such a precise alignment becomes increasingly unlikely. Truncating the eccentricity to be between 0.7 and 0.8 gives a probable mass of $24 M_{\text {Jup }}$ and a probable period of $13.3 \mathrm{yr}$. One conclusion with these eccentric orbits is that the ring system is within the Hill sphere for most of its orbit, but at periastron the Hill sphere shrinks down below the size of the outermost rings, providing a challenge for investigations into the stability of this ring system.

\section{CONCLUSIONS}

We interpret the 56-day J1407 light-curve event centered on UT 2007 April 30 as being due to a highly structured ring system surrounding an unseen secondary companion, supporting the conclusions in Mamajek et al. (2012), van Werkhoven et al. (2014), and Kenworthy et al. (2015). Using the gradients in the light curve generated by the finite size of the primary star, we solve for the geometry and impact parameter of the secondary companion and ring system. The size of the ring system is considerably larger than the Roche radius for the secondary companion and fills a significant fraction of the Hill radius. This implies that this structure is in a transitional state, with the rings at large radii undergoing accretion to form exosatellites orbiting the secondary companion. The rapid variation in ring density as a function of radius implies dynamical clearing processes that keep material out of the ring plane. Two such processes are (1) the formation of exomoons that are gravitationally clearing out these gaps, such as those sculpting the A-ring gaps in Saturn, and (2) the presence of Lindbad resonances, caused by the presence of unseen exosatellites at larger radii. For a secondary companion mass of $28 M_{\mathrm{Jup}}$, we interpret one of the most welldefined ring gaps at $6.1 \times 10^{7} \mathrm{~km}$ with a width of $4 \times 10^{6} \mathrm{~km}$ to be cleared by a satellite with an upper mass of $<0.8 M_{\oplus}$. This satellite would have an orbital period of $\sim 2 \mathrm{yr}$ in the ring system about J1407b.

We estimate the mass of the individual rings by assuming a dust opacity of $\kappa \sim 0.02 \mathrm{~cm}^{2} \mathrm{~g}^{-1}$ for unity optical depth, as assumed in Mamajek et al. (2012) for their estimate. The mass of each model ring is calculated without any correction for the projected inclination of the rings and is then plotted as the open circles in Figure 6. The mass of the rings is dependent on the orbital parameters of $\mathrm{J} 1407 \mathrm{~b}$, the effective dust opacity in the rings, the detailed dust size distribution, and the unknown ring structure where there is no photometric constraint. In the regions of no photometry, the ring structure is extended with the same optical properties at that at the closest known edge. The order-of-magnitude estimate for the total mass of the rings is $\sim 100 M_{\text {Moon }}$, close to the mass of the Earth. The ratio of the mass of the densest rings around Saturn (the B ring system) to that of the largest satellite, Titan, is approximately 1/4000. A large fraction of the mass is therefore accreted in the satellites. It is interesting to note that the satellite in J1407b has a similar mass 
(to an order of magnitude) as the rings, implying that further accretion into satellites is ongoing. For the cases where there are significant gaps in the photometric coverage or there is a window function imposed by the diurnal cycle, degeneracies appear in the fitted exoring models. Photometric data that continuously sample the eclipse light curve can completely solve the geometry of the exoring system.

Ring systems are thought to occur around other exoplanets, although none have been confirmed. Fomalhaut $\mathrm{b}$ is a comoving companion to the nearby $400 \mathrm{Myr}$ old star, moving on an eccentric orbit (Kalas et al. 2013; Mamajek 2012). A large ring system around the planet is thought to cause the anomalously bright flux seen in optical images. The orbit for $\beta$ Pictoris $\mathrm{b}$ is close to edge-on (Nielsen et al. 2014), indicating that it might transit its parent star. Anomalous photometry of $\beta$ Pictoris in 1981 (Lecavelier Des Etangs et al. 1995) has also been attributed to an extended system of material surrounding the planet, and the anomalous photometry extends over 30 days, implying that there is material extending out to 0.1 of the Hill radius of the planet. This could plausibly be caused by a giant ring system transiting $\beta$ Pictoris, similar to the ring system about J1407b.

With simple assumptions on ring geometry and the ring plane orientation, this ring model reproduces many but not all of the nightly photometric light curves. These discrepancies imply an error in the determined geometry of the ring plane and/or that the rings are not coplanar. Further modeling with additional degrees of freedom for the rings, such as warping and precession, may lead to better fits to the photometric data.

$\mathrm{J} 1407$ is currently being monitored both photometrically and spectroscopically for the start of the next transit. A second transit will enable a wide range of exoring science to be carried out, from transmission spectroscopy of the material to Doppler tomography that can resolve ring structure and stellar spot structure significantly smaller than that of the diameter of the star. The orbital period of J1407b is on the order of a decade or possibly longer. Searches for other occultation events are now being carried out (Quillen et al. 2014), and searches through archival photographic plates (e.g., DASCH; Grindlay et al. 2012) may well yield several more transiting ring system candidates.

We would like to acknowledge the anonymous referee for their careful reading of our paper and their comments, which have improved it. We have used data from the WASP public archive in this research. The WASP consortium comprises the University of Cambridge, Keele University, University of Leicester, the Open University, the Queen's University Belfast, St. Andrews University, and the Isaac Newton Group. Funding for WASP comes from the consortium universities and from the UK's Science and Technology Facilities Council. E.E.M. acknowledges NSF award AST-1313029.

Facility: SuperWASP

\section{REFERENCES}

Allard, F., Homeier, D., \& Freytag, B. 2012, RSPTA, 370, 2765

Armitage, P. J. 2011, ARA\&A, 49, 195

Barnes, J. W., \& Fortney, J. J. 2004, ApJ, 616, 1193

Braga-Ribas, F., Sicardy, B., Ortiz, J. L., et al. 2014, Natur, 508, 72

Butters, O. W., West, R. G., Anderson, D. R., et al. 2010, A\&A, 520, L10

Canup, R. M., \& Ward, W. R. 2002, AJ, 124, 3404

Claret, A., \& Bloemen, S. 2011, A\&A, 529, A75

Dong, S., Katz, B., Prieto, J. L., et al. 2014, ApJ, 788, 41

Dunham, D. W., Dunham, J. B., Binzel, R. P., et al. 1990, AJ, 99, 1636

Elliot, J. L., Dunham, E., \& Mink, D. 1977, Natur, 267, 328

Graczyk, D., Mikołajewski, M., Tomov, T., Kolev, D., \& Iliev, I. 2003, A\&A, 403, 1089

Graczyk, D., Soszyński, I., Poleski, R., et al. 2011, AcA, 61, 103

Grindlay, J., Tang, S., Los, E., \& Servillat, M. 2012, in IAU Symp. 285 , New Horizons in Time-domain Astronomy, ed. E. Griffin, R. Hanisch, \& R. Seaman (Cambridge: Cambridge Univ. Press), 29

Guinan, E. F., \& Dewarf, L. E. 2002, in ASP Conf. Ser. 279, Exotic Stars as Challenges to Evolution, ed. C. A. Tout \& W. van Hamme (San Francisco, CA: ASP), 121

Hamilton, C. M., Herbst, W., Vrba, F. J., et al. 2005, AJ, 130, 1896

Kalas, P., Graham, J. R., Fitzgerald, M. P., \& Clampin, M. 2013, ApJ, 775, 56

Kenworthy, M. A., Lacour, S., Kraus, A., et al. 2015, MNRAS, 446, 411

Kipping, D. M., Bakos, G. Á., Buchhave, L., Nesvorný, D., \& Schmitt, A. 2012, ApJ, 750, 115

Kipping, D. M., Hartman, J., Buchhave, L. A., et al. 2013, ApJ, 770, 101

Kley, W., \& Nelson, R. P. 2012, ARA\&A, 50, 211

Kloppenborg, B., Stencel, R., Monnier, J. D., et al. 2010, Natur, 464, 870

Lecavelier Des Etangs, A., Deleuil, M., Vidal-Madjar, A., et al. 1995, A\&A, 299,557

MacMahon, P. A. 1908, MNRAS, 69, 126

Magni, G., \& Coradini, A. 2004, P\&SS, 52, 343

Mamajek, E. E. 2012, ApJL, 754, L20

Mamajek, E. E., \& Bell, C. P. M. 2014, MNRAS, 445, 2169

Mamajek, E. E., Quillen, A. C., Pecaut, M. J., et al. 2012, AJ, 143, 72

McCarthy, D. W., Hubbard, W. B., Kulesa, C. A., et al. 2008, AJ, 136, 1519

Mikolajewski, M., Galan, C., Gazeas, K., et al. 2005, Ap\&SS, 296, 445

Mikolajewski, M., \& Graczyk, D. 1999, MNRAS, 303, 521

Millis, R. L., Wasserman, L. H., \& Birch, P. V. 1977, Natur, 267, 330

Nielsen, E. L., Liu, M. C., Wahhaj, Z., et al. 2014, ApJ, 794, 158

Pollacco, D. L., Skillen, I., Collier Cameron, A., et al. 2006, PASP, 118, 1407

Press, W. H., Teukolsky, S. A., Vetterling, W. T., \& Flannery, B. P. 1992, in Numerical Recipes in C. The Art of Scientific Computing (Cambridge: Cambridge Univ. Press)

Quillen, A. C., Ciocca, M., Carlin, J. L., Bell, C. P. M., \& Meng, Z. 2014, MNRAS, 441, 2691

Rattenbury, N. J., Wyrzykowski, Ł., Kostrzewa-Rutkowska, Z., et al. 2015, MNRAS, 447, 31

Shevchenko, V. G., \& Tedesco, E. F. 2006, Icar, 184, 211

Snellen, I. A. G., Brandl, B. R., de Kok, R. J., et al. 2014, Natur, 509, 63

Stewart, P. N., Tuthill, P. G., Hedman, M. M., Nicholson, P. D., \& Lloyd, J. P. 2013, MNRAS, 433, 2286

Tiscareno, M. S. 2013, in Planetary Rings, ed. T. D. Oswalt, L. M. French, \& P. Kalas (Dordrecht: Springer), 309

Tusnski, L. R. M., \& Valio, A. 2011, ApJ, 743, 97

van Werkhoven, T. I. M., Kenworthy, M. A., \& Mamajek, E. E. 2014, MNRAS, 441,2845

Verbiscer, A. J., Skrutskie, M. F., \& Hamilton, D. P. 2009, Natur, 461, 1098

Ward, W. R., \& Canup, R. M. 2010, AJ, 140, 1168

White, N. M. 1987, VA, 30, 13

Williams, J. P., \& Cieza, L. A. 2011, ARA\&A, 49, 67

Winn, J. N., Hamilton, C. M., Herbst, W. J., et al. 2006, ApJ, 644, 510 